\title{
Introduction to the special issue of Brain Structure and Function on transgenic modeling of neurodegenerative disorders
}

\author{
Patrick R. Hof · Gregory A. Elder
}

Published online: 19 February 2010

(C) Springer-Verlag 2010

\begin{abstract}
Animal models are widely used to study the pathogenesis of human diseases as well as to develop and test therapeutic strategies. Transgenic approaches now dominate animal modeling of human neurodegenerative diseases. This issue of Brain Structure and Function brings together a collection of peer-reviewed articles that illustrate the variety of ways in which transgenic modeling is being applied to neurodegenerative diseases. The articles range from systematic reviews of specific topics to articles that present original research findings and illustrate the methods of analysis that can be applied to transgenic models.
\end{abstract}

\section{P. R. Hof ( $\square)$}

Department of Neuroscience, Mount Sinai School of Medicine, One Gustave L. Levy Place, New York, NY 10029, USA

e-mail: patrick.hof@mssm.edu

\section{P. R. Hof}

Department of Geriatrics and Palliative Care,

Mount Sinai School of Medicine, One Gustave L. Levy Place, New York, NY 10029, USA

\section{P. R. Hof}

Computational Neurobiology and Imaging Center,

Mount Sinai School of Medicine, One Gustave Levy Place, New York, NY 10029, USA

\section{G. A. Elder}

Neurology Service, James J. Peters Department of Veterans Affairs Medical Center, 130 West Kingsbridge Road, Bronx, NY 10468, USA

\section{G. A. Elder}

Department of Psychiatry, Mount Sinai School of Medicine, One Gustave L. Levy Place,

New York, NY 10029, USA

G. A. Elder

Department of Neurology, Mount Sinai School of Medicine, One Gustave L. Levy Place, New York, NY 10029, USA
Gama Sosa et al. review the technologies that are available for producing transgenic animals in a variety of vertebrate and invertebrate species with an emphasis on the use of pronuclear injection and embryonic stem (ES) cell technology in the mouse. They also survey recent advances in how gene expression in transgenic animals can be modified through methods such as retroviral-mediated gene transfer, RNAi-mediated gene knockdown, and somatic cell mutagenesis combined with nuclear transfer and approaches that may allow transgenic animals to be created in species, where pronuclear injection and ES cell technology have been problematic.

A series of papers then discuss the application of transgenic technology in the mouse to a range of neurodegenerative disorders, including Alzheimer's disease (AD), the human tauopathies, amyotrophic lateral sclerosis, and familial British and Danish dementias. Crews et al. discuss the current understanding of AD pathophysiology. They then review the development of $\mathrm{AD}$ mouse models based on expression of mutant forms of the amyloid precursor protein (APP) that have been associated with familial $\mathrm{AD}(\mathrm{FAD})$ in humans, emphasizing in particular the role that toxic amyloid beta protein $(\mathrm{A} \beta)$ oligomers are thought to play in axonal and synaptic damage, as well as results suggesting that FAD mutants alter hippocampal neurogenesis. Elder et al. review what has been learned from the study of presenilin FAD mutant mice. Although these mice do not form plaques, they exhibit a number of central pathological features of $\mathrm{AD}$ including age-related neuronal and synaptic loss. The relevance of presenilin conditional knockout mice as models of $\mathrm{AD}$ is also discussed.

Three papers present primary research data illustrating how transgenic models can be used to study the pathological as well as electrophysiological basis of $\mathrm{AD}$ and 
human tauopathies. Takahashi et al. analyze, using stereologic methods, the regional pattern of age-related loss of parvalbumin, and calretinin immunoreactive neurons in the hippocampus of presenilin- 1 FAD mutant knockin mice as well as APP/PS1 transgenic mice. They find losses of both classes of neurons that are similar to the losses observed in human AD. Dickstein et al. discuss the human tauopathies in which abnormal accumulation of the microtubule-associated protein tau into neurofibrillary tangles (NFTs) is a defining pathological feature, diseases that include AD, the frontotemporal dementias, and progressive supranuclear palsy. Using quantitative three-dimensional morphometric methods, they show that with aging, overexpression of human tau in the mouse results in accumulation of aggregates of phosphorylated tau in neurons with associated changes in dendritic arborization and spine morphology, alterations that may provide a morphologic substrate for the loss of synaptic integrity and associated cognitive decline. Luebke et al. illustrate a multidimensional approach to understanding neurodegeneration that includes morphologic three-dimensional reconstructions, electrophysiological data acquired using confocal laser scanning microscopy, and whole-cell patch-clamp recording techniques combined with computational modeling. They show how these approaches can be applied to transgenic APP and tau models.

Wisniewski and Boutajangout provide a general review of current thinking on the pathogenesis of familial and sporadic $\mathrm{AD}$, and provide an overview of transgenic mouse models that exhibit amyloid or tau pathology. They then describe the exciting area of immunomodulation as a therapeutic strategy in $\mathrm{AD}$ and human tauopathies and the important role that mouse models have played in bringing this approach to human trials. However, they note the discordance between the encouraging results in the transgenic mouse models and the generally discouraging results of human clinical trials to date, observations that may suggest both limitations of the current mouse models as well as uncertainties in our understanding of the underlying pathogenesis of $\mathrm{AD}$.

Chen et al. report primary research findings using a transgenic mouse model of ALS that expresses a mutant superoxide dismutase-1 ( $m S O D 1)$ gene associated with familial ALS. They report that upregulation of nitric oxide synthase activity (iNOS or NOS2) occurs early in motor neurons and later in other cell types. They also report that inhibitors of iNOS delay the onset of disease and extend the lifespan of $m S O D 1$ mice. Familial British dementia (FBD) and familial Danish dementia (FDD) are neurodegenerative diseases caused by autosomal dominant mutations in the Bri2 gene. Both disorders exhibit parenchymal amyloid deposits, cerebral amyloid angiopathy, and neurofibrillary tangles. Garringer et al. review the current status of attempts to generate FDD models in the mouse by overexpressing wild-type and mutant forms of the Bri2 protein or by creating Bri2 knock-in mice, attempts that to date have been partially successful in reproducing the neuropathology of human FDD in the mouse.

The final group of articles discusses the use of three powerful model systems that are being increasingly used to model human neurodegenerative diseases. Iijima-Ando and Iijima summarize transgenic modeling of $\mathrm{AD}$ and tauopathies in Drosophila melanogaster, emphasizing the power of the genetic systems available that allow large-scale genome-wide screens for genetic modifiers to be performed. They review the insights that have come from the study of $\mathrm{A} \beta$ and tau neurotoxicity in the fly and how genetic modifiers of $\mathrm{A} \beta$ and tau toxicity have been identified. Ewald and Li discuss how the nematode Caenorhabditis elegans can be used to address the biological roles of the APP family proteins by examining the function of the $C$. elegans APPrelated gene, apl-1. They also discuss how components of the $\gamma$-secretase complex are being identified and their function discovered through genetic analyses in C. elegans as well as how transgenic $C$. elegans can be used to address the toxicity of different forms of the human $\mathrm{A} \beta$ peptide and tau protein. Sager et al. discuss the use of zebrafish, a highly versatile transgenic system that has rapidly developed in recent years. Highlighted are the structural and neurochemical similarities between the mammalian and zebrafish nervous systems and the many mammalian genes of interest to neurodegenerative diseases that are conserved in zebrafish. They describe the array of techniques for experimental manipulation and analysis of zebrafish that have been developed and review recent studies in which transgenic zebrafish have been used to model human tauopathies, polyglutamine diseases, and ALS.

Collectively, the articles presented in this issue of Brain Structure and Function illustrate the wide scope of transgenic modeling and illustrate many of the ways in which transgenic modeling is being applied to neurodegenerative diseases. These models have already improved our understanding of disease pathogenesis and led to new therapeutic approaches. They will without doubt continue to play central roles for years to come in both preclinical testing and as tools for developing insight into the biological basis of human neurodegenerative diseases. 